\title{
Experimentally Research on Stability Power of Erbium-doped Fiber Super Fluorescent Source
}

\author{
Xiaobo Zhou \\ Department of Electronic Information Engineering, Xijing University, Xi'an, China \\ 369146317@qq.com
}

Keywords: Super fluorescent fiber source (SFS); Power /Temperature control; Driving current; Optic power

\begin{abstract}
In order to obtain stability power of erbium-doped fiber super fluorescent source, the influence factors of temperature and power changes on the source are theoretically analyzed, according to the working principle and characteristics of SFS designed the driving circuit including automatic power control(APC) circuit and temperature control circuit which based on Peltier effect, experiment with $980 \mathrm{~nm}$ laser diode as pumping source, erbium-doped fiber erbium-doped concentration to $700 \mathrm{PPM}$, cutoff wavelength is $853.5 \mathrm{~nm}$, peak at $980 \mathrm{~nm}$ absorption coefficient is $4.5 \mathrm{~dB} / \mathrm{m}$, optimized erbium-doped fiber length $9 \mathrm{~m}$. The testing results show that the single pass forward super fluorescent source is very linear and stable by this driving circuit, and the stability is superior to $0.5 \%$, solved the problem of power instability.
\end{abstract}

\section{掺铒光纤超荧光光源功率稳定性实验研究}

周晓波

西京学院 电子信息工程系, 中国 陕西西安 710123

369146317@qq.com

摘要: 为了获得输出功率稳定的光纤超荧光光源, 理论分析了温度和功率对光源的影响, 根据 超荧光光源的工作原理和特性, 设计制作了自动功率控制（APC）驱动电路, 并应用帕尔帖效 应设计制作了基于 MAX1968 的温度控制电路, 实验采用 980nm 激光二极管作抽运光源, 掺铒光 纤的掺铒浓度为 $700 \mathrm{ppm}$, 截止波长为 $853.5 \mathrm{~nm}$, 在 $980 \mathrm{~nm}$ 处的峰值吸收系数为 $4.5 \mathrm{~dB} / \mathrm{m}$, 经 过优化设计选定长度为 $9 \mathrm{~m}$ 。经实验测定, 单程前向光纤超荧光光源在该自动功率控制电路和 温度控制电路驱动下获得了很好的线性度，且解决了功率不稳定问题，其稳定度优于 $0.5 \%$, 达到了较好的稳流效果。

关键词：超荧光光源；功率/温度控制；驱动电流；光功率

\section{1. 引言}

光源作为电信号转化为光信号的转化设备, 是光纤传感系统中关键的光学元件之一, 主要应 用的光源有表面光发射二极管 (LED)、激光二极管 (LD)、超辐射二极管 (SLD) 和超苂光光源 (SFS) [1-4]，而超苂光光源因其带宽宽、使用寿命长、输出功率高等优点成为研究的热点。 SFS (Super fluorescent Fiber Source) 利用掺稀土元素（如 Er3+、Nd3+、Yb3+等）光纤放大的 自发辐射 (Amplified Spontaneous Emission, ASE) 原理产生超苂光, SFS 在光纤传感系统、信 号处理、光学层析和医疗等领域具有广泛的应用 [5-8]。驱动电路驱动葲浦激光器, 百浦激光 器的稳定性决定着 SFS 输出功率的大小和稳定性, 进而直接影响着光纤传感器的准确度, 因 而研制高性能的驱动电路显得尤为重要 [9]。根据光源的工作原理和特性, 设计了自动功率控 制（APC）驱动电路和基于 MAX1968 的温度控制电路, 解决了功率不稳定问题, 实现了恒 温控制。 


\section{2. 原浦光源控制电路的设计}

激光器组件处于工作状态时, 若超过一定温度则会受到损伤, 因而洜浦光源的控制电路主要 包括 LD 驱动电路和保证 LD 安全工作的控制电路。

2. 1 . 自动温度控制电路的设计

激光二极管的许多关键参数（激射波长、阈值电流和效率等）都与二极管的结温有着密切的 关系, 且温度与二极管的寿命密切相关, 据估计, 每 $30^{\circ} \mathrm{C}$ 的温升会使寿命下降一个数量级 [10]:

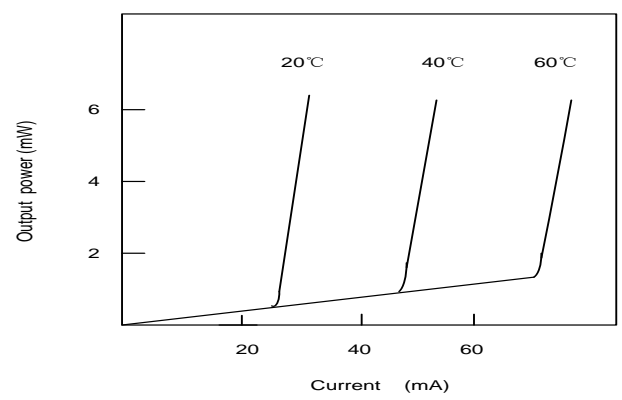

图 1 输出功率与电流关系图

由图 1 可以看到, 阈值电流随温度升高而明显增大, 当驱动电流小于阈值电流时, 输出光功 率近似为 0 , 超过阈值电流后, 光功率随驱动电流的增加呈线性上升趋势。在实际应用中, 希望激光二极管有尽可能低和稳定的温度, 为保证高精度地控制温度, 实验中选用易于控制、 启动快、体积小、结构简单、便于与 LD 封装的 TEC 作为温度控制的制冷器件, 它利用帕尔 贴效应进行电热转换装置，具有双向制冷和制热的能力。

MAX1968 是适用于 Peltier TEC 模块的开关型驱动芯片, 主要由两个开关型同步降压稳压器 组成, 能够提供 $\pm 3 \mathrm{~A}$ 双极性输出, 直接电流驱动和双向操作实现无死区的温度控制。两个稳 压器同时产生一个差动电压, 直接控制 TEC 电流, 实现电流的双向控制, 外部控制电路的输 出电压加在 TEC 电流控制输入端 CTL1 直接设置 TEC 电流, 流过 TEC 的电流由下式确定:

$$
I_{T E C}=\frac{V_{C T L I}-V_{R E F}}{10 R_{\text {SENSE }}}
$$

式中: $R_{S E N S E}$ 为 TEC 电流的感应电阻; $V_{C T L I}$ 为外部控制电路的输出电压; $V_{R E F}$ 为参考电压 (1.5V)。

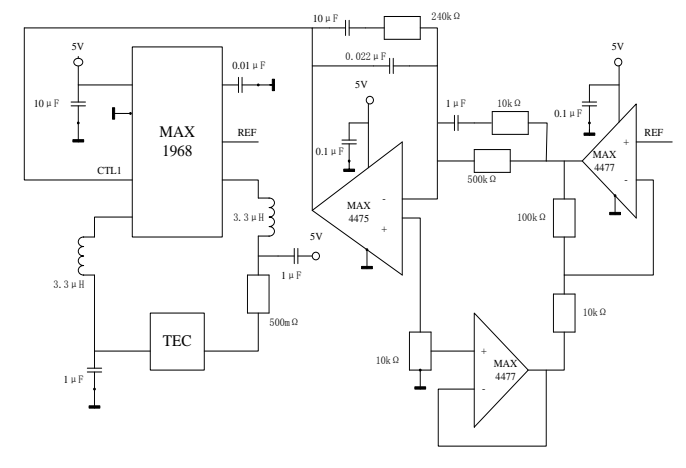

图 2 自动温度控制电路图

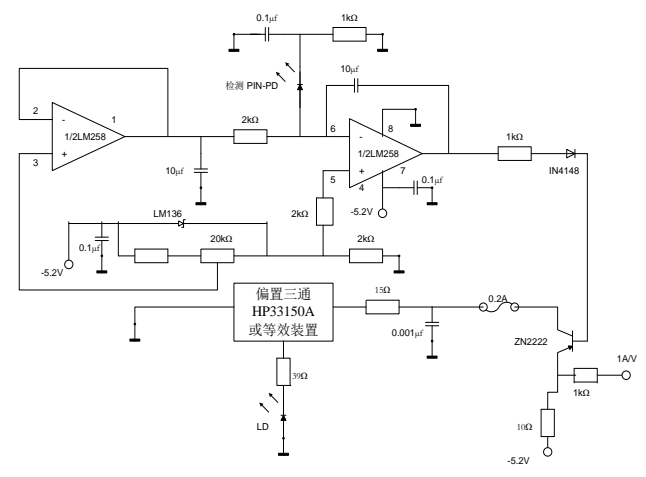

图 3 自动功率控制电路

基于 MAX1968 芯片的工作原理, 设计了如图 2 所示的自动温度控制电路, 该电路的工作原 理为: 温度传感器所提供的反馈信号与设定的温度值比较后得到的误差项经过放大处理送给 控制电路。常用的控制电路是由分立元件所构成的模拟 PID (比例、积分、微分) 控制器, 也可 以是数字 PID 控制, 但因为数字 PID 容易在系统中引入噪声, 所以需要进行适当的处理, 否 则会影响系统的性能。 


\section{2. 自动功率控制电路的设计}

要使泵浦激光器输出光强具有稳定性, 就要实现恒功率控制。利用半导体激光二极管内部封 装的功率监控器 (光电探测器), 把光电探测器监控到的激光输出的光功率变化, 作为反馈信 号, 控制 LD 的偏置电流, 使之进行相应的变化, 自动调节激光二极管工作电流的大小, 从 而使激光二极管的激光功率输出恒定。

半导体激光器自动功率控制电路图如图 3 所示。其工作原理如下: 当半导体激光器所发出的 激光强度变强时, 流过光电二极管 $\mathrm{PD}$ (装在激光二极管的内部) 的光电流随着增加, 加在减法 运算器反相端的电压也随之升高。经过减法运算放大器电路处理后输出电压减小; 减小的电 压信号经由放大器放大后加在了电压电流转换型恒流电路上, 并把它作为电压电流转换型恒 流电路的基准电压 $\mathrm{Ed}$, 运算放大器 $\mathrm{UA}$ 对取样电阻 $\mathrm{R}$ 上的压降与基准电压 $\mathrm{Ed}$ 进行比较, 其 微小差别将被放大, 并通过控制晶体三极管的基极电流的减小来控制流过半导体激光器的电 流, 使之变小, 从而使激光二极管发出的激光变弱。反之, 当激光二极管输出的激光强度变 弱时, 经过上述反馈网络以及控制电路, 同样将使激光二极管的输出激光变强。这样该电路 就形成了一个闭环系统, 最终使半导体激光器的输出光功率保持恒定。

\section{3. 实验结果及分析}

实验采用 $980 \mathrm{~nm}$ 激光二极管作抽运光源, 利用光功率计测量㬌浦源尾纤输出功率, 最大输出 功率为 $140 \mathrm{~mW}$, 中心波长为 $979.04 \mathrm{~nm}$, 允许最大抽运电流为 $300 \mathrm{~mA}$ 。所用的掺铒光纤掺铒浓 度为 $700 \mathrm{ppm}$, 截止波长为 $853.5 \mathrm{~nm}$, 在 $980 \mathrm{~nm}$ 处的峰值吸收系数为 $4.5 \mathrm{~dB} / \mathrm{m}$, 经过优化设计 选定长度为 $9 \mathrm{~m}$ 。光谱仪采用 AND0 公司生产的 AQ6319 型光谱仪进行输出光谱的纪录, 该光谱 仪最小分辨率为 $0.01 \mathrm{~nm}$, 测量范围为 50nm-2250nm。功率计为 JW3203CR 型手持式功率计, 其 校准波长可为 $850 \mathrm{~nm} 、 980 \mathrm{~nm} 、 1310 \mathrm{~nm} 、 1550 \mathrm{~nm}$, 功率测量范围为 $-40 \mathrm{dBm}-+200 \mathrm{dBm}$, 不确定 度为土 $5 \%$ 。

实验中搭建的掺饵光纤苂光光源结构图如图 4 所示:

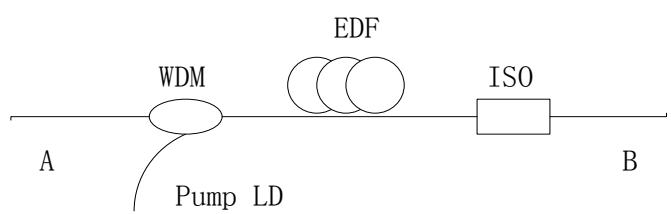

图 4 超荧光光源结构图

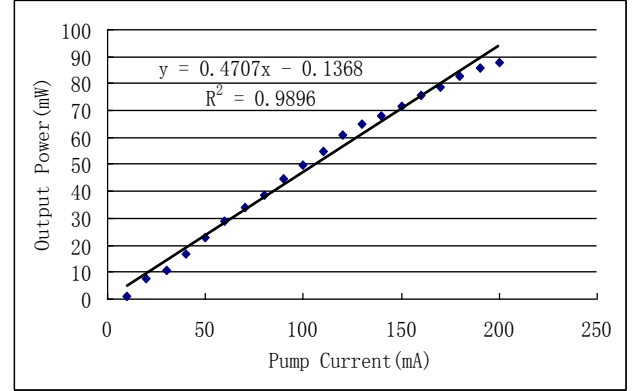

图 5 驱动电流与输出功率实验曲线

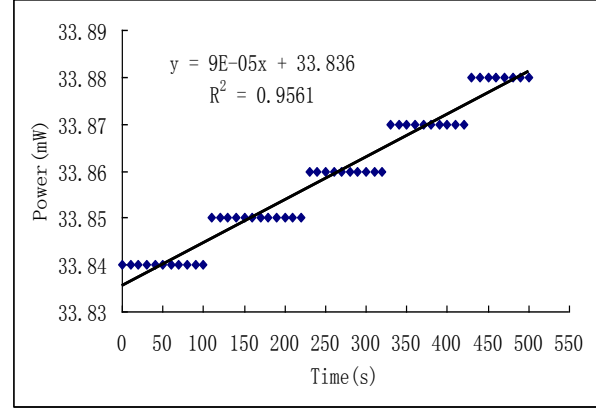

图 6 输出功率随时间拟合曲线

图 5 是在室温 $\left(25^{\circ} \mathrm{C}\right.$ ) 下, 用光功率计测得的激光二极管尾纤输出功率随抽运电流（从阈值 电流点起）变化图, 其基本呈线性关系, 由图中显示的激光器的 P-I 关系可以看出, 在 0-250 $\mathrm{mA}$ 的电流调节范围内, 该光纤传感器 $\mathrm{LD}$ 光源在所设计的电路驱动下, 输出功率与驱动电流在 大于阈值电流后拟合度高达 0.9896 , 呈现很好的线性关系, 即激光器的线性度良好。

图 6 是在室温 $\left(25^{\circ} \mathrm{C}\right)$ 下, 当输入电流恒定 $(70 \mathrm{~mA})$, 每隔 $100 \mathrm{~s}$ 测一组功率数值, 每组 10 个 
数据, 其时间间隔为 $10 \mathrm{~s}$, 共测得 5 组数据, 由国家标准 (GB/T 13864-92) 制定的激光辐射 功率稳定度测试方法中的公式 $S=\frac{\Delta P}{2 \times P_{a v}} \times 100 \%$ (S 为输出功率的稳定度, $\Delta P$ 为同一组中输 出功率最大值与最小值之差, $P_{a v}$ 为该组输出功率的平均值) 分析得到功率的控制精度优于 0. 5\%, 功率的稳定性良好。

\section{4. 结论}

对激光二极管的特性等多个方面进行了详细的分析, 并在此基础上设计制作了自动功率控制 电路和基于帕尔帖效应原理的自动温度控制电路, 设计方法简单、可靠、容易实现。高精度 的温控电路使激光器在预设的温度下能够稳定的工作, 保证了输出功率的稳定性。在室温 $\left(25^{\circ} \mathrm{C}\right)$ 下, 温度控制精度达到 $0.01^{\circ} \mathrm{C}$, 获得了优于 $0.5 \%$ 的功率控制精度, 达到了较好的稳 流效果。

致谢

陕西省教育厅专项基金 (2013JK1121); 西京学院科研基金 (XJ120235); 西京学院科研基金 (XJ140236)。

\section{参考文献}

[1] 张力, 刘承香, 阮双琛, 等. 高功率高效率掺铒光纤超苂光光源 [J]. 激光与光电子学进展, 2012,49(6):604.

[2] 陈倚竹, 张海涛, 巩马理, 等. 高平均波长稳定性超荧光光纤光源 [J]. 激光技术, 2014, 38(1):70-75.

[3] 吴旭, 刘承香, 张力, 等.双程前向结构掺铒光纤光源稳定性的实验研究 [J]. 中国惯性技 术学报, 2010,18(1):106-110.

[4] 吴旭, 刘承香, 阮双琛, 等.双程后向结构掺铒光纤光源的实验研究 [J]. 中国惯性技术学 报, 2012,20(6):720-724.

[5] Suo Xinxin, Yang Yuanhong, Yang Minwei, et al. High stability and radiation-resistance broadband fiber-optical source [C] //Proc. Of SPIE.2011:78533Z1-5.

[6] Hall D C, Burns W K, Moeller R P. High stability Er-doped superfluorescent fiber sources [J]. Lightwave Technol, 1995, 13(7):1452-1460.

[7] $\mathrm{Wu} \mathrm{Xu,} \mathrm{Ruan} \mathrm{Shuangchen,} \mathrm{Liu} \mathrm{Chengxiang,} \mathrm{et} \mathrm{al.} \mathrm{High-stable} \mathrm{Double-pass} \mathrm{Forward}$ Superfluorescent Fiber Source based on Erbium-doped Photonic Crystal Fiber[J].Applied Physics B:Lasers and Optics,2014,114(3):433-438.

[8] Wang Qi, Zhao Yong. Output power characteristics of C+L band erbium-doped superfluorescent fiber source [J]. Microwave and Optical Technology Letters, 2011, 53(10):2212-2215.

[9] Liu Chengxiang, Zhang Li, Wu Xu, et al. High-stability superfluorescent source based on Er3+-doped Photonic Crystal Fiber[J].Chinese Physic Letter,2012,29(6):064202.

[10]伍翔, 王一超, 冯重熙, 等.一种光输出功率控制电路[J].光通信研究, 2000,1:37-41.

\section{Acknowledgement}

Shaanxi Department of Education Special Research Project (2013JK1121); Xijing University Scientific Research Foundation Project (XJ120235) and Xijing University Scientific Research 
Foundation Project (XJ140236).

\section{References}

[1] Zhang Li, Liu Chengxiang, Ruan Shuangchen, et al. High-Power, High Efficiency Erbium-Doped Superfluorescent Fiber Source [J]. Laser \& Optoelectronics Progress, 2012, 49(6):604.

[2] Chen Yizhu, Zhang Haitao, Gong Mali, et al. A Superfluorescent Fiber Source with High mean wavelength stability[J].Laser Technology, 2014, 38(1):70-75.

[3] $\mathrm{Wu} \mathrm{Xu}$, Liu Chengxiang, Zhang Li, et al. Experimental investigation on Erbium-doped fiber source in double-pass forward configuration [J].Journal of Chinese Inertial Technology, 2010,18(1):106-110.

[4] Wu Xu, Liu Chengxiang, Ruan Shuangchen, et al. Experimental investigation on Erbium-doped fiber source with Double-Pass Backward Configuration [J]. Journal of Chinese Inertial Technology, 2012, 20(6):720-724.

[5] Suo Xinxin, Yang Yuanhong, Yang Minwei, et al. High stability and radiation-resistance broadband fiber-optical source [C] // Proc. Of SPIE.2011:78533Z1-5.

[6] Hall D C, Burns W K, Moeller R P. High stability Er-doped superfluorescent fiber sources [J]. Lightwave Technol, 1995, 13(7):1452-1460.

[7] $\mathrm{Wu} \mathrm{Xu}$, Ruan Shuangchen, Liu Chengxiang, et al. High-stable Double-pass Forward Superfluorescent Fiber Source based on Erbium-doped Photonic Crystal Fiber[J].Applied Physics B:Lasers and Optics,2014,114(3):433-438.

[8] Wang Qi, Zhao Yong. Output power characteristics of C+L band erbium-doped superfluorescent fiber source [J]. Microwave and Optical Technology Letters, 2011, 53(10):2212-2215.

[9] Liu Chengxiang, Zhang $\mathrm{Li}, \mathrm{Wu} \mathrm{Xu}$, et al. High-stability superfluorescent source based on Er3+-doped Photonic Crystal Fiber [J]. Chinese Physic Letter, 2012, 29(6):064202.

[10]Wu Xiang, Wang Yichao, Feng Chongxi, et al. Optical output power control in optical communication [J].Study on Optical Communications, 2000,1:37-41.

作者简介: 周晓波 (1982-), 女, 陕西西安人, 讲师, 主要从事光纤传感、光纤通信及光电 子学等领域的教学与研究工作, 369146317@qq. com 